\title{
Neutron-induced capture cross sections via the surrogate reaction method
}

\author{
M. Aïche ${ }^{1, a}$, G. Boutoux ${ }^{1}$, B. Jurado ${ }^{1}$, G. Barreau ${ }^{1}$, N. Capellan ${ }^{1}$, I. Companis ${ }^{1,8}$, S. Czajkowski $^{1}$, \\ D. Dassié ${ }^{1}$, B. Haas ${ }^{1}$, L. Mathieu ${ }^{1}$, A. Bail ${ }^{2}$, E. Bauge $^{2}$, J.M. Daugas ${ }^{2}$, T. Faul ${ }^{2}$, L. Gaudefroy ${ }^{2}$, \\ V. Méot $^{2}$, P. Morel ${ }^{2}$, N. Pillet ${ }^{2}$, O. Roig ${ }^{2}$, J. Taieb ${ }^{2}$, C. Théroine ${ }^{2}$, O. Sérot ${ }^{3}$, F. Gunsing ${ }^{4}$, J.T. Burke ${ }^{5}$, \\ X. Derkx ${ }^{6}$, and L. Tassan-Got ${ }^{7}$ \\ 1 CENBG, CNRS/IN2P3, Chemin du Solarium, BP. 120, 33175 Gradignan, France \\ 2 CEA - DAM DIF, 91297 Arpajon, France \\ 3 CEA Cadarache, DEN/DER/SPRC/LEPh, 13108 Saint Paul-lez-Durance, France \\ 4 CEA Saclay, DSM/DAPNIA/SPhN, 91191 Gif-sur-Yvette, France \\ 5 LLNL, US Department of Energy, Livermore, California 94551, USA \\ 6 Grand Accélérateur National d'Ions Lourds, Bld. Henri Becquerel, BP. 55027, 14076 Caen, France \\ 7 Institut de Physique Nucléaire, 15 rue Georges Clemenceau, 91406 Orsay, France \\ 8 HHNIP and Nuclear Engineering, PO Box MG-6, 077125 Bucharest-Magurele, Romania
}

\begin{abstract}
The surrogate reaction method is an indirect way of determining cross sections for nuclear reactions that proceed through a compound nucleus. In this method, the compound nucleus is produced via an alternative (surrogate) reaction and its decay (by fission, gamma or neutron emission) is measured in coincidence with the outgoing appropriate charged particle. This technique has enabled neutron-induced cross sections to be extracted for nuclear reactions on short-lived nuclei that otherwise could not be measured. The CENBG collaboration has successfully applied this technique to determine the neutron-induced fission cross sections of several short-lived nuclei such as ${ }^{233} \mathrm{~Pa},{ }^{242,243} \mathrm{Cm}$ and ${ }^{241} \mathrm{Am}$. These data are very important for the development of the $\mathrm{Th} / \mathrm{U}$ cycle and for minor actinide transmutation. We currently investigate whether this powerful technique can also be used to determine the neutron-induced capture cross sections. For this purpose we will use the surrogate reaction ${ }^{174} \mathrm{Yb}\left({ }^{3} \mathrm{He}, \mathrm{p} \gamma\right){ }^{176} \mathrm{Lu}$ to infer the well known ${ }^{175} \mathrm{Lu}(\mathrm{n}, \gamma)$ cross section and compare the results with the directly measured neutron-induced data. The experimental set-up and the first results will be presented. We will also discuss our future plans to use the surrogate method for extracting actinides $(\mathrm{n}, \gamma)$ cross sections.
\end{abstract}

\section{Introduction}

Neutron-induced cross sections of short-lived nuclei are of great interest in applied science and fundamental physics. However, very often such cross sections are very difficult to measure directly due to the fabrication issues on account of the radioactive decay of the targets. These difficulties can be overcome with the so called surrogate reaction technique, developed in the 1970's by Cramer and Britt [1]. It consists of obtaining neutron induced cross-sections by measuring the decay probability of a compound nucleus (e.g., fission, neutron emission, or radiative capture) produced via an alternative (surrogate) reaction. In this work the surrogate reaction used is a few nucleon transfer reaction, as illustrated in Figure 1.

\footnotetext{
a e-mail: aiche@cenbg.in2p3.fr
}

This is an Open Access article distributed under the terms of the Creative Commons Attribution-Noncommercial License 3.0, which permits unrestricted use, distribution, and reproduction in any noncommercial medium, provided the original work is properly cited. 


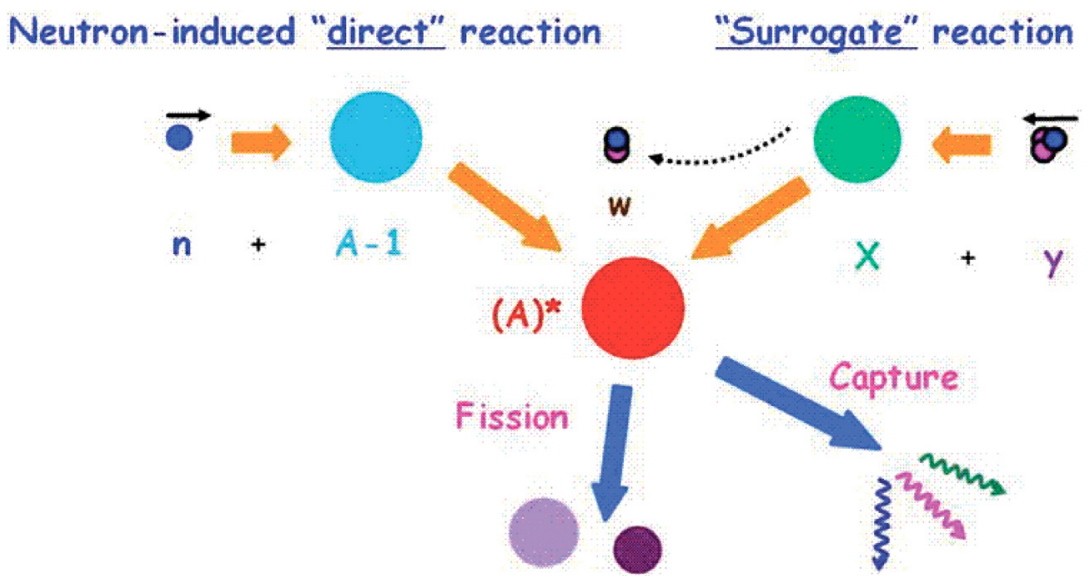

Fig. 1. Schematic representation of the surrogate reaction method. The surrogate reaction is here a transfer reaction $\mathrm{X}(\mathrm{y}, \mathrm{w}) \mathrm{A}^{*}$. Three possible exit channels (fission, $\gamma, \mathrm{n} \gamma$ ) are also represented.

The left part of Figure 1 represents the desired neutron-induced reaction on the target A-1, which leads to the compound-nucleus $\mathrm{A}$ at an excitation energy $\mathrm{E}^{*}$. The nucleus A can decay through different exit channels: fission, neutron emission, etc... On the right part of Figure 1, in the surrogate reaction method, the same compound nucleus $\mathrm{A}$ is produced by a transfer reaction between a projectile $\mathrm{y}$ (a light charged particle) and a target $\mathrm{X}$. In the exit channel of the transfer reaction $(\mathrm{y}+\mathrm{X} \rightarrow \mathrm{A}+\mathrm{w})$ we have the heavy recoil nucleus $\mathrm{A}$ and an outgoing particle w (proton, deuton, triton, etc). The identification of the ejectile $\mathrm{w}$ permits to determine the mass $\mathrm{A}$ and charge $\mathrm{Z}$ of the decaying nucleus. In addition, we can deduce the excitation energy $\mathrm{E}^{*}$ of the compound nucleus $\mathrm{A}$ by measuring the kinetic energy and the emission angle of the ejectile w. Experimentally, the detection of the ejectile w, in coincidence or not, with a given decay product (e.g., fission fragments or gammas) of the compound nucleus $A^{*}$ gives the decay probability $P_{\text {decay }}^{A \text { exp }}\left(E^{*}\right)$ of the given reaction channel. According to the surrogate reaction method, the measurement of the fission or capture probability permits to determine the neutron-induced cross section for the nucleus A-1 as follows:

$$
\sigma_{\text {decay }}^{A-1}\left(E_{n}\right)=\sigma_{C N}^{A}\left(E_{n}\right) \cdot P_{\text {decay }}^{A, \exp }\left(E^{*}\right)
$$

where $\sigma_{C N}^{A}\left(E_{n}\right)$ is the compound nucleus formation cross section in the desired reaction (formation of the nucleus $\mathrm{A}$ after a neutron absorption with an energy $\left.\mathrm{E}_{\mathrm{n}}\right)$. In our case, $\sigma_{C N}^{A}\left(E_{n}\right)$ is obtained from the optical model calculations performed with the TALYS code [9]. One of the biggest interest of the surrogate method is that in some cases the targets needed are less radioactive than the target A-1 of the desired reaction.

An important issue to be investigated in the context of surrogate reactions is the difference between the distributions in spin and parity $(\mathrm{J}, \pi)$ of the states populated in the desired and surrogate reactions, a problem referred to as the $\mathrm{J}^{\pi}$ population mismatch in the litterature [2]. Since the $\mathrm{J}^{\pi}$ population influences the decay probabilities of the compound nucleus, one would expect differences between the decay probabilities measured in surrogate and in neutron induced experiments. However, in the Weisskopf-Ewing limit, the decay probability is independent of the spin and parity of the compound nucleus and eq. 1 is valid. The conditions under which the Weisskopf-Ewing limit applies have been investigated in Refs [3]. This limit holds when the excitation energy is sufficiently high for the decay widths to be dominated by the statistical level density. Neutron induced fission cross sections of ${ }^{233} \mathrm{~Pa}$ [12], ${ }^{242} \mathrm{Cm}$ and ${ }^{243} \mathrm{Cm}$ [13] have been obtained by the CENBG collaboration with this technique (see Ref. [4]). The agreement observed at the lowest energies between the surrogate results and the neutron-induced data indicates that the $(\mathrm{J}, \pi)$ distributions populated with both reaction mechanisms are quite similar. In this contribution, we focus on capture cross section measurements which, 
compared to fission, may be more sensitive to the difference of spin-parity distributions populated in neutron-induced and surrogate reactions. Our aim is to study the transfer reaction ${ }^{174} \mathrm{Yb}\left({ }^{3} \mathrm{He}, \mathrm{p}\right){ }^{176} \mathrm{Lu}$ as surrogate for the ${ }^{175} \mathrm{Lu}(\mathrm{n}, \gamma)$ cross sections. We have considered the ${ }^{175} \mathrm{Lu}(\mathrm{n}, \gamma)$ cross section since it presents the advantage to be one of the best known capture cross sections, see for example $[5,6]$. Lutetium isotopes have attract considerable interest in astrophysics due to their importance to s-process studies but also in the interpretation of radiochemical activation in the inertial confinement of fusion experiments.

\section{Capture probability of ${ }^{175} \mathrm{Lu}$}

\subsection{Experimental set-up}

We performed the ${ }^{174} \mathrm{Yb}\left({ }^{3} \mathrm{He}, \mathrm{p}\right)$ measurement at theTandem accelerator at the IPN Orsay in March 2010. We used an energy of $24 \mathrm{MeV}$ for the incident ${ }^{3} \mathrm{He}$ ions beam with an intensity of $20 \mathrm{nA}$. To infer the capture probability, gammas rays are detected in coincidence with the ejectiles. The latter are fully identified by two large area $\Delta \mathrm{E}$-E telescopes placed symmetrically at $130^{\circ}$ with regard to the ${ }^{3} \mathrm{He}$ beam. The E detectors are two $\mathrm{Si}(\mathrm{Li}) 3000 \mu \mathrm{m}$ detectors. The $\Delta \mathrm{E}$ detectors are two $300 \mu \mathrm{m}$ silicon position sensitive detectors, whose $16^{*} 16 \mathrm{X}-\mathrm{Y}$ strips [7] provide the angle of the detected ejectile with an angular coverage of $108^{\circ}$ to $152^{\circ}$. Notice that we shield the Si detectors with an electrostatic Mylar(Al) screen made of $3 \mu \mathrm{m}$ negatively biased at $300 \mathrm{~V}$ in order to prevent the interaction of thermal electrons with the telescopes. We use a set of four $\mathrm{C}_{6} \mathrm{D}_{6}$ liquid scintillators for gamma detection. The total number of gamma cascades can be obtained with these detectors by using the total energy detection principle in combination with the pulse height weighting technique. The advantage of this technique is that it allows one to determine the gamma-cascade detection efficiency independently of the level scheme of the decaying nucleus. The same technique was used to obtain the ${ }^{233} \mathrm{~Pa}(\mathrm{n}, \gamma)$ cross section [8]. Notice that the coupling of these detectors to a pulse shape discriminator permits to separate between photons and neutrons interacting with the scintillators. The ${ }^{174} \mathrm{Yb}$ target is also surrounded by six germanium detectors. They are used to measure low-lying-ray transition intensities as a function of the compound nucleus excitation energy, which is an experimental way to investigate the difference in spin distributions between transfer and neutron-induced reactions. Figure 2 illustrates our experimental set-up.

\subsection{Preliminary data analysis}

The identification of the light charged particles and the determination of their energy and scattering angle is achieved in the Si detectors. With this information and the related Q-values, the excitation

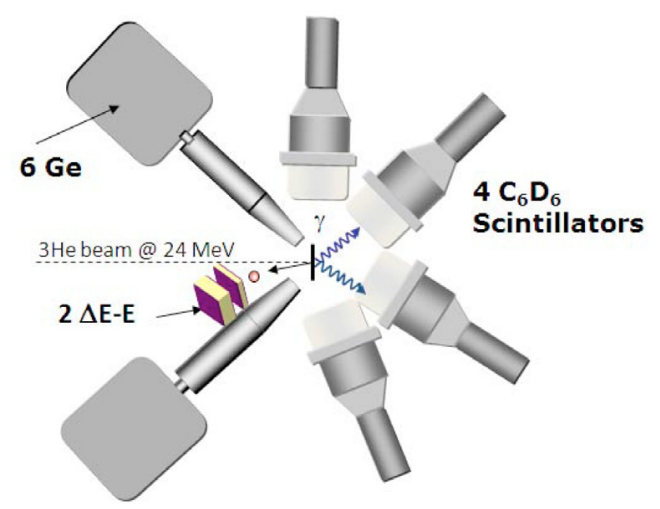

Fig. 2. Experimental set-up @ TANDEM IPNOrsay. 


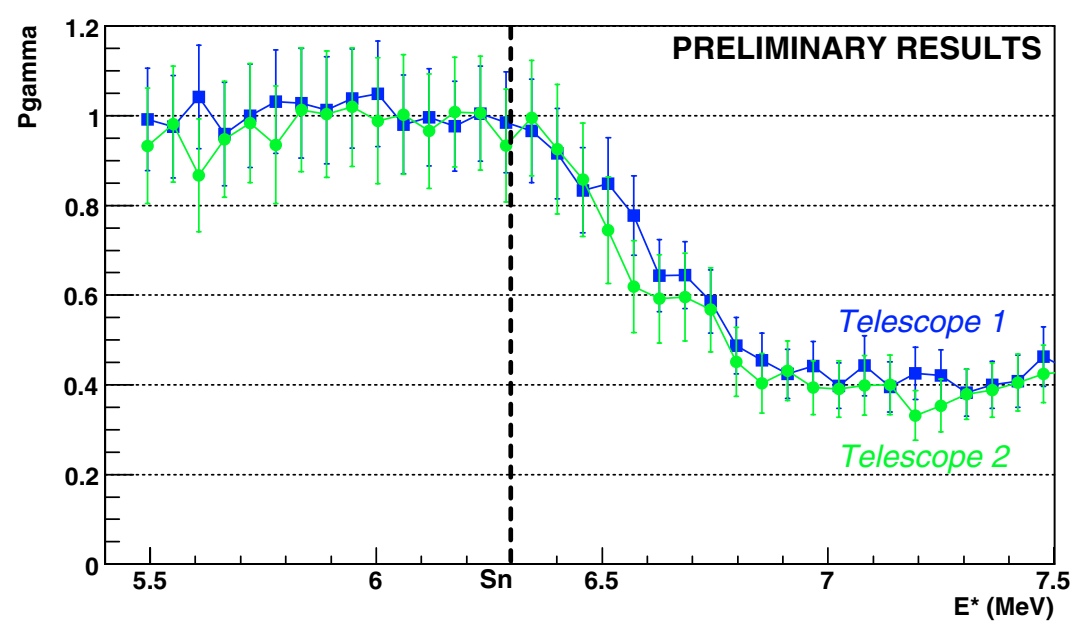

Fig. 3. Preliminary results: Experimental $\mathrm{P}_{\gamma}$ for the neutron-induced radiative capture of ${ }^{175} \mathrm{Lu}$.

energy $\mathrm{E}^{*}$ of the corresponding compound nucleus is determined. The experimental radiative capture probability $\mathrm{P}_{(n ; \gamma)}\left(\mathrm{E}^{*}\right)$ of ${ }^{176} \mathrm{Lu}$ can be obtained in the following way:

$$
P_{n, \gamma}\left(E^{*}\right)=N_{\text {coinc }}\left(E^{*}\right) /\left(N_{\text {single }}\left(E^{*}\right) \cdot \varepsilon_{\gamma}\left(E^{*}\right)\right)
$$

where $\varepsilon_{\gamma}$ represents the C6D6 photon detection efficiency, $\mathrm{N}_{\text {coinc }}\left(\mathrm{E}^{*}\right)$ are the number of protons detected in coincidence with one of the $\mathrm{C}_{6} \mathrm{D}_{6}$ detectors and $\mathrm{N}_{\text {single }}\left(\mathrm{E}^{*}\right)$ the total number of protons, i.e. the number of ${ }^{176 \mathrm{~L}} \mathrm{u}$ formed. Since the nucleus ${ }^{176} \mathrm{Lu}$ is formed by a transfer reaction, it is possible to extend our investigation below neutron separation energy $S_{\mathrm{n}}$ corresponding to $E^{*}=6.29 \mathrm{MeV}$ where only $\gamma$-rays can be emitted and consequently the measured emission probability should be 1 (see Fig. 3).

Below the neutron separation threshold, the ratio $\mathrm{N}_{\text {coinc }}\left(\mathrm{E}^{*}\right) / \mathrm{N}_{\text {single }}\left(\mathrm{E}^{*}\right)$ gives the total $\mathrm{C}_{6} \mathrm{D}_{6}$ detectors efficiency. This ratio is of around $15 \%( \pm 3 \%)$ and remains constant from $\mathrm{E}^{*}=5.5 \mathrm{MeV}$ to $\mathrm{S}_{\mathrm{n}}$. Assuming that this preliminary value for the efficiency independent of excitation energy also above $S_{n}$ we obtain the probability for emitting a gamma cascade of ${ }^{176} \mathrm{Lu}$ as a function of $\mathrm{E}^{*}$ (Figure 3). Our data are compared with a TALYS calculation for the neutron-induced capture probability of ${ }^{175} \mathrm{Lu}$ [9]. The parameters of the code have been tuned to reproduce the experimental data for the ${ }^{175} \mathrm{Lu}(\mathrm{n}, \gamma)$ cross sections. The preliminary results of Figure 3 show that our data agree rather well with the calculation right above the neutron separation energy. Nevertheless, our data are clearly above the capture probability for $\mathrm{E}^{*}>6.5 \mathrm{MeV}$. This is caused by the opening of the neutron-inelastic decay channel $\left(n^{\prime} \gamma\right)$, which has not yet been substracted from our data. Our aim is to determine the efficiency for detecting a gamma cascade independently by using the weighting function technique, which requires

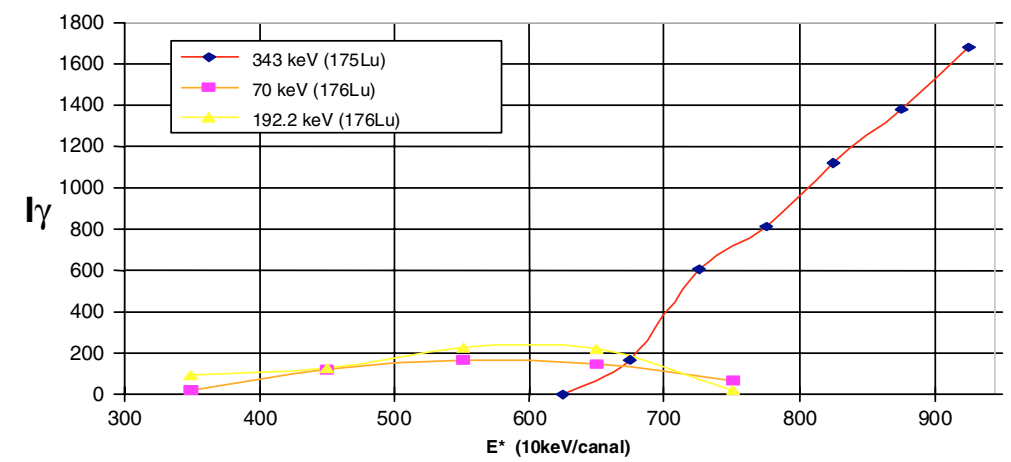

Fig. 4. $\gamma$-rays intensities for $\left({ }^{3} \mathrm{He}, \mathrm{p}()\right.$ and $(3 \mathrm{He}, \mathrm{pn}$ '() reactions versus the excitation energy of $176 \mathrm{Lu}$. 
complete knowledge of the response functions for the $\mathrm{C}_{6} \mathrm{D}_{6}$ detectors for a large range of gamma energies. These have been determined experimentally using gamma-ray sources, inelastic scattering reactions and transfer reactions on light nuclei, as explained in [10]. Simulations, using MCNP 4C2 and GEANT4 transport codes, permit to build response functions of the $\mathrm{C}_{6} \mathrm{D}_{6}$ detectors for unmeasured gamma energies. This work remains to be done.

On the other side, Germanium detectors are used for the detection of discrete low-lying $\gamma$-rays emitted by the residual nucleus in coincidence with the proton ejectile. This information will be useful to constrain reaction deexcitation models and infer the calculated level spin distribution. The latter requires modelling the decay of the compound nucleus and fitting the relevant parameters of the spin distribution to reproduce the experimental data. In addition, $\gamma$-ray intensity ratios of isomeric states can be measured. This observable is very sensitive to the spin distribution and has the advantage that it can be directly compared to the same quantity measured in a neutron-induced experiment. The population of the $\left(\mathrm{n}^{\prime} \gamma\right)$ low-lying state of the ${ }^{175} \mathrm{Lu}$ in the present measurements is nicely illustrated by the disappearance of the $\gamma$ transition $5 / 2+\rightarrow 7 / 2+343 \mathrm{keV}$ at $\mathrm{E}^{*}=\mathrm{Sn}=6.29 \mathrm{MeV}$ (Fig. 4).

Finally, the use of germanium detectors will also considerably help in the identification of the decaying nucleus. The prompt and delayed Germanium spectra observed experimentally are in good agreement with previous studies [11]. As mentioned above, with C6D6 detectors alone it is not possible to disentangle between a gamma arising from the $(3 \mathrm{He}, \mathrm{p})$ and from the $(3 \mathrm{He}, \mathrm{pn})$ reaction. Statistical model calculations are used to correct for this effect. However, with the Germaniums we can measure excitation functions for certain low-lying transitions related to the (3He,pn) reaction. These excitation functions represent a precious piece of information for improving the models used to correct data of Figure 3 for this effect.

\section{Conclusion and perspectives}

We have performed an experiment to study the validity of the surrogate method for extracting capture cross sections. We have used the well known ${ }^{175} \mathrm{Lu}(\mathrm{n}, \gamma)$ cross section to test the ${ }^{174} \mathrm{Yb}\left({ }^{3} \mathrm{He}, \mathrm{p}\right)$ reaction. Our experimental set-up uses $\mathrm{C}_{6} \mathrm{D}_{6}$ scintillators for determining the capture probability. The use of germanium detectors will allow us to extract information on the spin-parity distribution of the populated states. Ge detectors will also be useful in order to correct the measured gamma probability from ${ }^{174} \mathrm{Yb}\left({ }^{3} \mathrm{He}, \mathrm{pn}\right)$ events. First preliminary results have been presented. The analysis of the other transfer channels $\left(\mathrm{d}, \mathrm{t}\right.$ and $\alpha$ ) will also allow us to extract the $(\mathrm{n}, \gamma)$ cross sections for ${ }^{174} \mathrm{Lu}(\mathrm{T} 1=2=3.31 \mathrm{y})$,

${ }^{173} \mathrm{Lu}(\mathrm{T} 1=2=1.37 \mathrm{y})$ and ${ }^{172} \mathrm{Yb}$. This experiment is extremely important in view of the application of the surrogate method to infer capture cross sections of minor actinides, which are crucial for nuclear waste incineration. In the future, we plan to use the ${ }^{237} \mathrm{~Np}(\mathrm{n}, \gamma),{ }^{238} \mathrm{U}(\mathrm{n}, \gamma)$ and ${ }^{232} \mathrm{Th}(\mathrm{n}, \gamma)$ cross sections to validate the ${ }^{238} \mathrm{U}\left({ }^{3} \mathrm{He}, \mathrm{t}\right){ }^{238} \mathrm{~Np},{ }^{238} \mathrm{U}(\mathrm{d}, \mathrm{p}){ }^{239} \mathrm{U}$ and ${ }^{232} \mathrm{Th}(\mathrm{d}, \mathrm{p}){ }^{233} \mathrm{Th}$ surrogate reactions respectively. Finally, we would like to stress that the surrogate approach is very general and in principle can be employed to determine cross sections for all types of compound-nuclear reactions on a large variety of nuclei and applications (such as astrophysics, nuclear fusion, etc ...).

This work was supported by the CNRS program PACEN/GEDEPEON and the EURATOM 6 Framework Programme European Facilities for Nuclear Data Measurements. (EFNUDAT), contract number FP6-036434.

\section{References}

1. J.D. Cramer, H.C. Britt, Nucl. Sci. and Eng., 41, 177 (1970)

2. J. Escher, F.S. Dietrich, Phys. Rev. C,74, 054601 (2006)

3. W. Younes, H.C. Britt, Phys. Rev. C,67, 024610 (2003)

4. B. Jurado et al., Conf. Proceedings of CNR*09, Bordeaux 2009, 06004 (2010)

5. K. Wisshak et al., Phys. Rev. C,73, 015807 (2006)

6. M. Heil et al., Nucl. Instr. Meth. A,459, 229 (2001)

7. S.N. Soisson et al., Nucl. Instr. Meth.A,doi:10.1016/j.,ima.2009.11.053 (2010) 


\section{EPJ Web of Conferences}

8. S. Boyer et al., Nucl. Phys. A,775, 175 (2006)

9. A.J. Koning, S. Hilaire, et al., User Manual Talys

10. J.N. Wilson et al., Nucl. Inst. Meth. A,511, 388 (2003)

11. W. Andrejtsche_et al., Nucl. Phys. A,226, 142 (1974)

12. M. Petit et al., Nucl. Phys. A,735, 345 (2004)

13. G. Kessedjian et al., Phys. Lett. B, Volume 692, 297-301 (2010) 